\title{
Anabases
}

ANABASES Traditions et réceptions de l'Antiquité

\section{Un pape biographe : Jésus de Nazareth de Joseph Ratzinger-Benoît XVI}

A Pope Biographer: Jesus of Nazareth by Joseph Ratzinger-Benedict XVI

\section{Philippe Foro}

\section{(2) OpenEdition}

1 Journals

Édition électronique

URL : http://journals.openedition.org/anabases/7978

DOI : 10.4000/anabases.7978

ISSN : 2256-9421

\section{Éditeur}

E.R.A.S.M.E.

\section{Édition imprimée}

Date de publication : 9 novembre 2018

Pagination : 253-264

ISSN : 1774-4296

\section{Référence électronique}

Philippe Foro, « Un pape biographe : Jésus de Nazareth de Joseph Ratzinger-Benoît XVI », Anabases [En ligne], 28 | 2018, mis en ligne le 09 novembre 2020, consulté le 20 janvier 2021. URL : http:// journals.openedition.org/anabases/7978; DOI : https://doi.org/10.4000/anabases.7978 


\title{
Un pape biographe : Jésus de Nazareth de Joseph Ratzinger-Benoît XVI
}

\author{
Philippe Foro
}

U

n pape biographe de Jésus de Nazareth, avouons que la situation n'est pas commune, voire inédite. C'est cette aventure spirituelle, théologique et exégétique que le pape Benoît XVI a entreprise en publiant trois volumes, consacrés à celui que les chrétiens considèrent comme le Christ, Messie et Fils de Dieu ${ }^{1}$. “Ouragan éditorial » selon la formule de Philippe Levillain à propos du premier d'entre eux ${ }^{2}$, cette œuvre est également une “ surprise » pour le monde

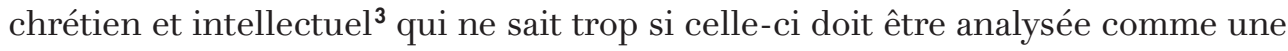
publication du Magistère ou d'un intellectuel catholique désireux de donner son interprétation de la vie et de la mission de Jésus ${ }^{4}$. Après avoir rapidement présenté une des personnalités les plus importantes du catholicisme contemporain, nous aborderons les raisons qui ont amené Joseph Ratzinger à entreprendre un tel travail, la manière dont il a analysé certains aspects de la vie de Jésus au travers de quelques exemples pris dans les trois volumes, la réception rencontrée par le dernier des grands opus de celui qui est désormais le pape émérite depuis le 28 février 2013.

1 J. Ratzinger - Benoît XVI, Jésus de Nazareth. Du baptême dans le Jourdain à la Transfiguration, Paris, 2007 ; Jésus de Nazareth. De l'entrée à Jérusalem à la Résurrection, Perpignan, 2011 ; L'enfance de Jésus, Paris, 2013.

2 Ph. Levillain, Le moment Benoît XVI, Paris, 2008, p. 267. Le premier volume s'est vendu à un peu plus d'un million d'exemplaires.

3 B. Lecomte, Benoît XVI, Paris, 2011 (1 éd. 2006), p. 150.

4 Sur ce point, Joseph Ratzinger-Benoît XVI a toujours expliqué qu'il n'avait pas écrit ce livre en tant que pape. Nous y reviendrons. 


\section{Prêtre, professeur, cardinal-archevêque et pape}

La carrière de Joseph Ratzinger au sein de l'Église est digne d'intérêt ${ }^{5}$. Né en 1927 à Marktl am Inn en Bavière, il est ordonné prêtre en 1951. Alors vicaire à Munich, il soutient, deux ans plus tard, son doctorat portant sur Peuple et Maison de Dieu dans la doctrine de l'Église chez Saint Augustin. Nommé professeur de théologie dogmatique et fondamentale à Freising, il présente une habilitation, en 1957, consacrée à La théologie de l'histoire chez Bonaventure. Joseph Ratzinger occupe ensuite des postes de professeur de théologie fondamentale à Bonn en 1959, de théologie dogmatique et d'histoire du dogme à Münster en 1963, à Tübingen en 1966, à Ratisbonne en 1969. Entre temps, il est conseiller du cardinal Frings, archevêque de Cologne de 1942 à 1969, lors du concile Vatican II dont il est un des experts. Le père Ratzinger est nommé archevêque de Munich par le pape Paul VI le 25 mars 1977, puis créé cardinal le 29 juin. Le 25 novembre 1981, Jean-Paul II lui confie la préfecture de la Congrégation pour la Doctrine de la Foi et la présidence de la Commission biblique pontificale. De 1986 à 1992, le cardinal Ratzinger coordonne les travaux de la commission du catéchisme de l'Église catholique. Doyen du Sacré Collège en 2002, il organise la période préparant au conclave qui suit le décès de Jean-Paul II. Le 19 avril 2005, il est élu pape et prend le nom de Benoît XVI, en l'honneur du fondateur de l'ordre bénédictin, père de l'Europe, et du pape Benoît XV, pris dans la tourmente de la Première Guerre mondiale.

\section{Pourquoi une biographie de Jésus ?}

Suite à la crise moderniste qui a sclérosé une partie de la recherche catholique dans la première moitié du xx siècle, l'encyclique Divino Afflante Spiritu publiée par le pape Pie XII en 1943 a incité à une nouvelle approche des textes des Écritures par l'utilisation de la critique textuelle. " Cet art de la critique textuelle, qu'on emploie avec beaucoup de succès et de fruit dans l'édition des textes profanes, doit servir aujourd'hui, à plus forte raison en vérité, pour les Livres Saints, à cause du respect qui est dû à la parole divine. Le but de cet art est, en effet, de restituer le texte sacré, autant qu'il se peut, avec la plus grande perfection, en le purifiant des altérations dues aux insuffisances des copistes et en le délivrant, dans la mesure du possible, des gloses et des lacunes, des inversions de mots et des répétitions, ainsi que des fautes de tout genre qui ont coutume de se glisser dans tous les écrits transmis à travers plusieurs siècles ${ }^{6}$. Le concile Vatican II affine la question de l'étude des textes bibliques dans la constitution Dei verbum du 18 novembre 1965 :

5 La dernière biographie en date est celle d'E. Guerriero, Serviteur de Dieu et de l'humanité: la biographie de Benoît XVI, Tours, 2017. Préfacée par le pape François, elle fait quelque peu figure de biographie officielle.

- PIE XII, Divino Afflante Spiritu, 30 septembre 1943. 
“Cependant, puisque Dieu, dans la Sainte Écriture, a parlé par des hommes à la manière des hommes, il faut que l'interprète de la Sainte Écriture, pour voir vraiment ce que Dieu lui-même a voulu nous communiquer, cherche avec attention ce que les hagiographes ont vraiment voulu dire et ce qu'il a plu à Dieu de faire passer par leurs paroles. Pour découvrir l'intention des hagiographes, on doit entre autres choses, considérer aussi les genres littéraires. Car c'est de façon bien différente que la vérité se propose et s'exprime en des textes diversement historiques, ou prophétiques, ou poétiques, ou même en d'autres genres d'expression. Il faut, en conséquence, que l'interprète cherche le sens que l'hagiographe, en des circonstances déterminées, dans des conditions de son temps et de sa culture, employant les genres littéraires alors en usage, entendait exprimer et a, de fait, exprimé. » ${ }^{7}$

Afin de comprendre le raisonnement intellectuel qui amène le pape à rédiger sa biographie de Jésus, le discours qu'il prononce, le 12 septembre 2008, aux Bernardins à Paris s'avère très utile :

“Sous de nombreux aspects, la situation actuelle est différente de celle que Paul a rencontrée à Athènes, mais, tout en étant différente, elle est aussi, en de nombreux points, très analogue. Nos villes ne sont plus remplies d'autels et d'images représentant de multiples divinités. Pour beaucoup, Dieu est vraiment devenu le grand Inconnu. Malgré tout, comme jadis où derrière les nombreuses représentations des dieux était cachée et présente la question du Dieu inconnu, de même, aujourd'hui, l'actuelle absence de Dieu est aussi tacitement hantée par la question qui Le concerne. Quaerere Deum - chercher Dieu et se laisser trouver par Lui : cela n'est pas moins nécessaire aujourd'hui que par le passé. Une culture purement positiviste, qui renverrait dans le domaine subjectif, comme non scientifique, la question concernant Dieu, serait la capitulation de la raison, le renoncement à ses possibilités les plus élevées et donc un échec de l'humanisme, dont les conséquences ne pourraient être que graves. Ce qui a fondé la culture de l'Europe, la recherche de Dieu et la disponibilité à L'écouter, demeure aujourd'hui encore le fondement de toute culture véritable. " ${ }^{8}$

La rédaction du premier volume débute pendant l'été 2003. Les quatre premiers chapitres sont rédigés à la fin du mois d'août 2004 et l'ouvrage est bien avancé mais non terminé lors du conclave d'avril 2005. Benoît XVI estime qu'il doit aller jusqu'au bout du projet malgré les charges qu'implique la charge pontificale. “ Après mon élection au siège pontifical de Rome, j’ai consacré tous mes instants de liberté à la rédaction de ce livre „ ${ }^{9}$. La préface du livre Jésus de Nazareth. Du baptême dans le Jourdain à la Transfiguration est signée le 30 septembre 2006. Les préfaces des deux autres volumes sont respectivement paraphées le 25 avril 2010

7 Concile Vatican II. Les documents. Dei verbum, Paris, Médiapaul, 2012, p. 141-142.

8 Site du Saint Siège consulté le 22 octobre 2017.

9 Ratzinger - Benoît XVI, Jésus de Nazareth, p. 19. 
et le 15 août 2012. Le temps employé à la rédaction des trois volumes a s'en doute empêché Benoît XVI de rédiger plus d'encycliques ${ }^{10}$. “ On peut y voir évidemment une mauvaise priorité. En tout cas, c'est l'une des raisons. Et puis aussi parce que, après la grande profusion d'encycliques que nous avait offerte Jean-Paul II, je pensais qu'il serait bon d'adopter un rythme plus lent " ${ }^{\prime \prime}$. Comment le pape travailla-t-il à la biographie de Jésus? Il s'en explique :

“ Pour commencer, il faut tout relire, tout méditer. D’une part en partant des textes de l'Évangile, d'autre part en dialoguant avec les principaux ouvrages d'exégèse. C'est une redécouverte. C'était aussi un progrès spirituel que de me replonger jusqu'aux fondements et d'être capable de le dire. Car ce n'est que lorsqu'on est capable d'exprimer les choses et de les dire qu'on les a comprises. „12

Une importante question est de savoir à quel titre l'auteur écrit cette biographie de Jésus. Pape, chef de l'Église catholique ou intellectuel parmi des intellectuels? Voici la réponse avancée par Benoît XVI :

“ Il ne s'agit pas d'un livre lié à ma fonction didactique, que j’aurais écrit en vertu de mon pouvoir pontifical. C'est un livre dont je m'étais proposé depuis longtemps de faire mon dernier grand opus et que j'ai déjà commencé à écrire avant d'être élu pape. Je ne voulais pas, et c'était tout à fait conscient, dispenser un enseignement officiel, mais participer aux confrontations théologiques et tenter de présenter une exégèse, une interprétation du Texte qui ne suive pas un historicisme positiviste mais intègre le foi comme élément de l'interprétation. Dans le paysage actuel de l'exégèse, il s'agit bien entendu d'un risque gigantesque. Mais, si l'interprétation des Textes sacrés veut vraiment être une théologie, il faut bien le prendre. Et si la foi doit nous aider à comprendre, elle ne doit pas être conçue comme un obstacle, mais comme une aide, afin que nous nous rapprochions des textes qui viennent de la foi et veulent y mener. "s 13

Il est certes difficile de séparer totalement dans l'esprit des lecteurs le pontife du simple intellectuel donnant sa perception de Jésus de Nazareth, d'autant plus que l'éditeur italien, puis les divers éditeurs qui ont proposé les ouvrages en plusieurs langues, ont voulu que le prénom pontifical de Benoît XVI soit accolé à Ratzinger, et cela est d'ailleurs souligné par une partie des réceptions de l'œuvre. Comme l'écrit Michel Deneken, prêtre et théologien, “ on peut, sans irrévérence, supposer que Ratzinger aurait suscité un lectorat bien moins nombreux et dans un

10 Benoît XVI publie trois encycliques durant son pontificat : Deux caritas est en 2005, Spe salvi en 2007, Caritas in veritate en 2009.

11 Benoît XVI, Dernières conversations avec Peter Seewald, Paris, 2016, p. 236.

12 Ibidem, p. 235.

13 Benô̂t XVI, Lumière du monde avec Peter Seewald, Paris, 2012, p. 225-226. 
cercle bien plus restreint que Benoît XVI „14. Nous aurons l'occasion d'y revenir. Voici l'analyse avancée par l'historien Paul Christophe :

" En le publiant sous son nom de famille et sous son nom de pape, il signifie qu'il y a travaillé avant son élection et que ce n'est « en aucune manière un acte du magistère ». C'est l'expression de sa recherche personnelle et par conséquent chacun est libre de le contredire. Cependant Benoît XVI ne le considère pas moins comme une œuvre importante. Car il y dénonce encore les présentations d’un Jésus «modernisé ou postmodernisé ", un Jésus réduit à n'être qu'un homme de son temps. Il refuse le fossé creusé entre le « Jésus historique » et le “ Christ de la foi ». Ce livre s'appuie sur l'exégèse historico-critique dont le pape souligne à la fois l'importance et les limites. Benoît XVI veut aller au-delà. Il présente son ouvrage comme un livre de croyant, une œuvre de « théologie biblique », une méditation sur le Jésus de la foi. Il se propose d'atteindre le sens plénier de l'Écriture pour faire connaître et comprendre Jésus à ses lecteurs et pour les inviter à l'aimer. „15

Benoît XVI s'en explique lui-même à plusieurs reprises lors d'entretiens avec le journaliste allemand Peter Seewald :

« Je tenais vraiment à le faire. En effet, de même que la liturgie occupe une place centrale dans l'expérience que l'Église a d'elle-même et que rien ne va plus quand la liturgie n'est plus elle-même, de même l'Église s'essouffle quand nous ne connaissons plus Jésus. Le risque est considérable qu'il soit détruit pour nous par certains types d'exégèses, qu'il s'épuise à force de discours. Il a donc fallu que je m'enfonce un peu dans une forêt de détails. Une analyse spirituelle du dogme ne suffisait pas ici. Il fallait prendre part à la querelle, sans se perdre dans les détails exégétiques mais avec suffisamment de profondeur pour constater que la méthode historique ne nous interdit pas la foi. $)^{16}$

Est-ce donc le rapport à l'histoire, ou plus précisément à l'exégèse historicocritique, qui a également motivé le cardinal, puis pape, à travailler à une biographie de Jésus? Lors du synode de Rome sur la parole de Dieu, Benoît XVI ne rejette pas le fait que l'histoire soit un élément d'appréhension de la foi chrétienne et du christianisme lui-même. Il déclare le 14 octobre 2008 : « Le fait historique est une dimension constitutive de la foi. L'histoire du salut n'est pas une mythologie, mais une véritable histoire, et c'est pour cela qu'elle doit être étudiée avec les méthodes

14 M. Deneken, « Le principe 'pour'. La christologie proexistentielle du Jésus de Nazareth de Benoît XVI. Un palimpseste du Seigneur de Guardini », Recherches de Science Religieuse, 99, 2011/4, p. 499-510, en part. p. 499.

15 P. Christophe, Benoît XVI, un pontificat contrasté, Paris, 2013, p. 28.

16 Benoît XVI, Dernières conversations, p. 234. 
de la recherche historique sérieuse. „ ${ }^{17} \mathrm{Il}$ est clair lors de la rédaction de la préface de son premier volume. La critique historique ne peut appréhender à elle seule la figure du Christ mais elle a eu son utilité même si elle a atteint ses limites.

“Les progrès de la recherche historico-critique ont débouché sur des distinctions de plus en plus subtiles entre les différentes strates de la tradition, au terme desquelles la figure de Jésus, à laquelle la foi se réfère nécessairement, devient de plus en plus floue, voire évanescente. Dans le même temps, les reconstitutions de Jésus, qui devaient être recherchées derrière les traditions des évangélistes et de leurs sources, sont apparues de plus en plus antinomiques: du révolutionnaire anti-romain, travaillant au renversement des pouvoirs en place et échouant, évidemment, dans cette entreprise, au doux moraliste, qui consent à tout et, du coup, finit par causer lui-même sa perte sans qu'on en comprenne très bien les raisons. Quand on fait une lecture comparée de plusieurs de ces reconstitutions, force est de constater qu'elles reflètent d'avantage leurs auteurs et leurs idéaux qu'elles ne mettent au jour l'icône du Christ, alors devenue floue. Par conséquent, la méfiance à l'endroit de ces différentes images de Jésus s'est incontestablement accrue, alors que la figure même de Jésus s'éloignait encore plus de nous. (...) Mais j'espère que le lecteur verra clairement que ce livre n'est pas écrit contre l'exégèse moderne, qu'il témoigne au contraire d'une grande reconnaissance pour tout ce qu'elle nous a donné et continue de nous donner. Elle nous a fait accéder à une abondance de matériaux et de connaissances qui présentent la personne de Jésus de façon bien plus vivante et bien plus profonde que nous ne pouvions l'imaginer il y a encore quelques décennies. „ ${ }^{18}$

Observons à présent quelques analyses de Joseph Ratzinger-Benoît XVI au travers d'épisodes significatifs de la vie de Jésus.

\section{Quelques analyses sur la vie et la mission de Jésus}

Il n'est pas question, dans le cadre de cette contribution, de faire une présentation exhaustive de la pensée de Joseph Ratzinger à propos de Jésus. Nous avons fait le choix de présenter trois analyses prises, non pas dans le cadre chronologique de la vie de Jésus, mais en suivant la publication des trois volumes consacrés à celle-ci par Benoît XVI. Tout d'abord, l'épisode de la Transfiguration relaté dans le chapitre 9 de l'évangile de Marc et le chapitre 17 de l'évangile de Matthieu. L'auteur met en présence des travaux exégétiques ayant réfléchi sur la Transfiguration. L'exégète dominicain Jean-Marie van Cangh (1942-2013) et l'exégète jésuite Michel van Esbroeck (1934-2003) mettent en relation la confession de foi de Pierre qui précède la Transfiguration avec la fête juive du Yom Kippour, et la Transfiguration elle-même avec Soukkhot, la fête des Tentes. Le jésuite Jean Daniélou (1905-1974) concentre l'épisode avec le seul lien avec la fête des Tentes.

17 Cité dans Petitfils, Jésus, p. 21.

18 Ratzinger-Benoît XVI, Jésus de Nazareth, p. 8 et 18-19. 
Hartmut Gese (1929), théologien protestant, estime que la Transfiguration du Christ est un écho avec la montée de Moïse au Sinaï au chapitre 24 de l'Exode. De cette diversité d'analyse, Joseph Ratzinger écrit que « la datation à partir du calendrier des fêtes juives (lui) paraît plus convaincante ” ${ }^{19}$. Mais il ajoute sa propre réflexion sur la signification de cette théophanie :

“Cette puissance (dýnamis) du Royaume leur (les apôtres Pierre, Jean et André présents à la Transfiguration) apparaît dans le Jésus transfiguré qui parle avec les témoins de l'Ancienne Alliance de la “ nécessité » de sa passion comme chemin vers la gloire (Luc, 24, 26-27). Ainsi ils voient l'anticipation de la Parousie, ainsi ils entrent progressivement dans la profondeur du mystère de Jésus. „20

Ensuite, la date de la Cène ${ }^{21}$. Selon les évangiles synoptiques (Marc, Matthieu, Luc) le dernier repas du Christ avec ses disciples se place un jeudi, veille de la Pâque. L'arrestation a lieu dans la nuit du jeudi au vendredi. Le procès et la mise à mort de Jésus se déroulent le vendredi. La résurrection s'effectue le dimanche. Le principal problème est de placer le procès et la mise à mort le jour de la Pâque juive ce qui paraît fort peu probable. Dans l'Évangile de Jean, le dernier repas entre Jésus et les Douze n'est pas un repas pascal. Celui-ci doit être le vendredi soir car les dignitaires juifs ne veulent pas entrer dans le prétoire de Pilate " pour ne pas se souiller mais pour pouvoir manger la Pâque »(Jean, 18, 22). Le vendredi est donc la veille de la Pâque. La mort de Jésus a lieu au moment de l'immolation des agneaux au Temple. Une tentative de concilier les deux chronologies est proposée par l'exégète Annie Jaubert (1912-1980)22. S'appuyant sur le livre des Jubilés, ouvrage juif de la seconde moitié du $\mathrm{II}^{\mathrm{e}}$ siècle avant J.-C. et la Didascalie des Apôtres du III $^{\mathrm{e}}$ siècle, la chercheuse française avance que la Pâque s'est tenue le mercredi et le repas pascal le mardi, l'arrestation dans la nuit du mardi au mercredi, le procès et l'exécution s'étalant les jeudi et vendredi. Joseph Ratzinger reconnaît à la démonstration une réelle érudition mais en contradiction avec une tradition remontant au II $^{\mathrm{e}}$ siècle et qui fait du jeudi, le jour de la dernière Cène. Aussi, ne lui est-il « simplement pas possible de l'accueillir ». Par contre, Benoît XVI se rallie à la position du père jésuite John Paul Meier, auteur d'une œuvre majeure sur Jésus en quatre volumes. Dans le premier d'entre $e^{23}{ }^{23}$, le père Meier se rallie à la chronologie johannique tout comme Benoît XVI qui en tire une conclusion théologique.

19 Ibidem, p. 336.

20 Ibidem, p. 346.

21 Ce dossier est exposé ibidem, p. 132-142.

22 A. Jaubert, La date de la Cène. Calendrier biblique et liturgie chrétienne, Paris, 1957.

23 J. P. Meier, Un certain Juif Jésus. Les données de l'histoire. Les sources, les origines, les dates, Paris, 2005, p. 247-253. 
“ Une chose est évidente dans toute la tradition : l'essentiel de cette Cène de congé n’a pas été la Pâque ancienne, mais la nouveauté que Jésus a réalisée dans ce contexte. Même si ce banquet de Jésus avec les Douze n’a pas été un repas pascal selon les prescriptions rituelles du judaïsme, en rétrospective la connexion intérieure de l'ensemble avec la mort et la résurrection de Jésus est apparue évidente : c'était la Pâque de Jésus. Et, en ce sens, il a célébré la Pâque et il ne l'a pas célébrée : les rites anciens ne pouvaient pas être pratiqués ; quand vint le moment, Jésus était déjà mort. Mais il s'était donné lui-même et ainsi il avait vraiment célébré la Pâque avec eux. De cette façon, l'ancien rite n'avait pas été nié, mais il avait seulement été porté ainsi à son sens plénier. „ ${ }^{24}$

Enfin, l'enfantement virginal de Jésus ${ }^{25}$. Après avoir rappeléles rapprochements effectués par des historiens des religions entre la conception et la naissance de Jésus et des naissances connues au sein des polythéismes (pharaons égyptiens, Hercule, Persée), Joseph Ratzinger observe que “ dans les récits des Évangiles, l'unicité du Dieu unique et l'infinie différence entre Dieu et la créature demeurent parfaitement conservées. Il n'existe aucune confusion, il n'y a aucun demi-dieu. Le Parole créatrice de Dieu, par elle-même, opère quelque chose de nouveau »²6. Puis, rejoignant le théologien protestant Karl Barth (1886-1968), il conclut que :

“Dans l'histoire de Jésus il y a deux moments dans lesquels l'action de Dieu intervient immédiatement dans le monde matériel : la naissance par la Vierge et la résurrection du tombeau, dans lequel Jésus n'est pas resté et n'a pas subi la corruption. Ces deux faits représentent un scandale pour l'esprit moderne. On concède à Dieu d'opérer sur les idées et les pensées, dans la sphère spirituelle, mais non dans la sphère matérielle. Cela dérange. Là n'est pas sa place. Mais il s'agit justement de cela : c'est-à-dire que Dieu est Dieu, et qu'il n'évolue pas seulement dans le monde des idées. (...) Mais il possède ce pouvoir et, par la conception et la résurrection de Jésus-Christ, il a inauguré une nouvelle création. Ainsi, en tant que Créateur, il est aussi notre Rédempteur. Pour cette raison, la conception et la naissance de Jésus de la Vierge Marie sont un élément fondamental de notre foi et un signal lumineux d'espérance. » ${ }^{27}$

\section{Réceptions de l'œuvre}

Il y a tout d'abord les enthousiastes. Peter Seewald, journaliste allemand qui a été l'interlocuteur de Benoît XVI pour la publication de Lumière du monde et Dernières conversations, est de ceux-là.

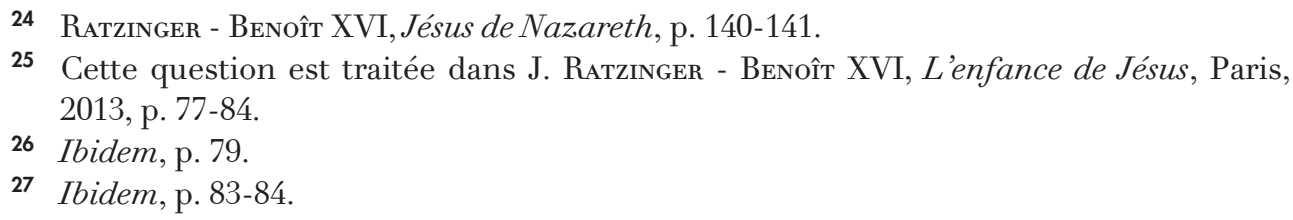


“L'ouvrage en trois volumes qu'il a consacré au Christ suffit à rendre ce pontificat unique. Benoît XVI a offert l'incontournable vade-mecum pour l'avenir de la théologie, de la catéchèse et de la formation des prêtres, bref, le fondement de la doctrine de la foi pour le troisième millénaire. Ce n'était pas dans une chaire universitaire mais bien dans celle de saint Pierre que la boucle pouvait être bouclée. Nul autre ne possédait la culture, les antécédents, la force et l'inspiration nécessaires pour restaurer avec une méticulosité scientifique et un réalisme mystique l'image de Jésus, égratignée à en être méconnaissable, et la rendre à l'humanité. „ ${ }^{28}$

C'est également le cas d'Alain Besançon, communiste ayant rompu avec le PCF en 1956, ancien directeur à l'École Pratique des Hautes Études et spécialiste de la Russie et de l'Union soviétique ; dans L'Osservatore Romano du 23 mars 2011, au moment de la sortie du deuxième volume, il écrit : « Le premier sentiment que j’ai éprouvé quand j'ai lu le livre a été d'admiration. J'ai de nombreuses raisons pour admirer ce livre, comme chrétien, comme catholique et enfin comme professeur. Je sais assez bien ce qu'est un bon livre. Celui-ci, formellement, est excellent, digne non seulement d'un cardinal et d'un pape (l'auteur signe avec ces deux titres), mais, et je le dis sans ironie, d'un grand maître ». S'appuyant sur les grands réformateurs du xvI siècle, Alain Besançon se fait aussi militant. "Quand je vois un livre qui ose dire que la Vierge Marie a eu plusieurs enfants, je le rejette avec la même indignation qu'éprouvaient Luther et Calvin quand une thèse similaire était soutenue devant eux. C'est l'incarnation qui en jeu ». Jean-Marie Guénois, chroniqueur religieux au Figaro apprécie l'œuvre, comme il l'écrit dans ce quotidien le 11 mars 2011. « On y retrouve la méticulosité du théologien allemand qui compare les versions des différents évangiles, les mettant en débat avec les points de vue, souvent contradictoires, des exégètes, ces spécialistes de la Bible. Benoît XVI coupe parfois les cheveux en quatre mais dessine, au final, un portrait inattendu du fondateur du christianisme ». Mais le journaliste insiste sur le fait que le travail du pape souligne que le message du Christ n'est pas, en premier lieu, une morale. “ C'est au fond un regard très intérieur sur le Christ qu'il appelle mais aussi critique sur les habitudes de pensée. En changeant les perspectives, Benoît XVI, pourtant très respectueux de la tradition, veut rompre avec l'image d'une Église catholique aliénante et cadenassée. »

Le théologien Michel Deneken, devenu président de l'université de Strasbourg en décembre 2016, met en avant la filiation intellectuelle de Benoît XVI avec le père Romano Guardini qui fut son professeur à Munich, auteur de Le Seigneur. Méditations sur la personne et la vie de Jésus-Christ, œuvre traduite en français en 1946. « Même relativisation de l'exégèse, même rejet d'une distinction systématique du Jésus de l'histoire et du Christ de la foi, même progression à partir de la structure 
narrative des évangiles, même approche théologique et spirituelle de l'Écriture, même refus d'érudition »29. Si Michel Deneken souligne les limites trouvées à la méthode historico-critique dans la compréhension de la parole biblique, il met en exergue l'attachement de Benoît XVI à l'exégèse canonique. “Se référant à Dei verbum 12, Benoît XVI conclut l'explication de cette deuxième orientation en montrant la pertinence de la méthode d'exégèse canonique, principe fondamental de l'exégèse catholique. Cette lecture canonique suppose que l'on considère que Jésus fait l'unité de l'un et de l'autre Testament. (...) L'unité de l'Écriture constitue une donnée de la foi et non le résultat de l'exégèse. » ${ }^{30}$

D’autres spécialistes sont plus réservés par les options méthodologiques du pape. Pour l'essayiste Viviane de Montalembert, la difficulté de séparer l'auteur de sa fonction pontificale est un élément clé. À propos du second volume, elle écrit que « nous avons affaire (...) à un livre de Benoît XVI qui fait comme s'il n'était pas Benoît XVI mais seulement Joseph Ratzinger. Il semble pourtant évident, dès l'avant-propos, que ses déclarations annonçant la fin de l'exégèse historico-critique comme seule méthode d'interprétation des textes bibliques n'acquièrent leur véritable poids que du fait que c'est la pape qui le dit. " ${ }^{31}$ Pierre Lassave, sociologue des religions à l'École des Hautes Études en Sciences Sociales, reconnaît sans difficulté que l'œuvre est servie par « une langue limpide et pédagogique » ainsi que par la richesse des ponts entre Ancien et Nouveau Testament. Néanmoins, " une double tension traverse donc cette entreprise herméneutique : entre théologie et histoire d'une part et entre réflexion personnelle et signature pontificale d'autre part. Sa réception hors des cercles immédiats de l'Église en a accusé les traits ambivalents, soit que pour les uns l'essai ait brillamment surmonté les obstacles, soit que pour les autres il n'ait pu les lever. Le second volume s'appuie sur les premiers encouragements pour aborder des questions aussi cruciales que celles de la passion et de la résurrection du Christ. „32 Mais la critique la plus importante est la volonté de l'auteur de s'éloigner des apports de la plus récente des exégèses :

“ L'aversion à peine voilée de l'auteur pour s'enquérir du contexte historique de la rédaction des Testaments, pour revenir sur les tribulations de leur transmission mettant aux prises des communautés concurrentes, pour dérouler le fil des interférences entre lois divines et règles humaines, dit assez que la théologie qu'il

29 M. Deneken, “ Le principe 'pour' », p. 500.

30 Ibid, p. 503.

31 V. De Montalembert, “ Jésus selon Joseph Ratzinger, pape », https://www.lacourdieu. com/a-propos.html?id=338, consulté le 5 décembre 2016.

32 P. Lassave, “ «Joseph Ratzinger-Benoît XVI, Jésus de Nazareth. Deuxième partie. De l'entrée à Jérusalem à la Résurrection ", Archives de sciences sociales des religions 156, octobre-décembre 2011, p. 263. 
prône s'éloigne de toute confusion avec l'histoire des religions. Pour cette dernière et plus généralement les sciences sociales des faits religieux, ce second essai christologique de Ratzinger-Benoît XVI constitue un document d'importance en ce qu'il marque, non sans talent, la distance prise par la hiérarchie catholique avec un certain savoir critique sur ses sources scripturaires. Étude des textes fondateurs à la lumière des disciplines universitaires que Vatican II avait pourtant nourrie de ses encouragements. „33

Hans Küng a également une observation distanciée de l'œuvre de Joseph Ratzinger. Il est vrai que la relation entre les deux hommes est une des aventures humaines et intellectuelles parmi les plus intéressantes du catholicisme contemporain. Né en 1928 en Suisse alémanique, ordonné prêtre en 1954, ayant soutenu une thèse sur La justification. La doctrine de Karl Barth. Réflexion catholique, Hans Küng devient professeur à Tübingen où il se lie avec un autre enseignant, Joseph Ratzinger. Théologien expert durant le concile Vatican II, il entame une opposition avec Rome qui lui vaut, en 1979, la suspension de son enseignement de théologie, même s'il reste à l'université de Tübingen avec une chaire de théologie œcuménique, créée pour lui par ses collègues ${ }^{34}$. Par la suite, il lui fut donné à plusieurs reprises de ferrailler avec la Congrégation pour la Doctrine de la Foi dont le préfet n'est autre que le cardinal Ratzinger depuis 1981. Hans Küng avait publié un ouvrage sur Jésus en 1978. Une réédition intervint en 2014, donc après la sortie des trois volumes de Joseph Ratzinger. Aussi, ne manqua-t-il pas l'occasion de réagir vis-à-vis de l'œuvre de son ancien collègue devenu pape :

“ On comparera mon livre sur Jésus avec les trois livres sur Jésus du pape émérite Benoît XVI-Joseph Ratzinger. Nous avons tous deux forgé notre conception de Jésus alors que nous étions professeurs de dogmatique à Tübingen dans les années 1960. Bien entendu, je ne veux créer aucune opposition irréductible entre nos deux conceptions. Mais il faut savoir que dès son livre sur La Foi chrétienne hier et aujourd'hui, datant de Tübingen, mon collègue Ratzinger présenta une caricature polémique des recherches modernes sur Jésus, tandis que j'entreprenais résolument de m'expliquer avec l'exégèse historique et critique et fondais ainsi mon livre $\hat{E}$ tre chrétien de façon systématique et rigoureuse sur le résultat de l'étude critique du Nouveau Testament. Tout en reconnaissant du bout des lèvres son acceptation de la méthode historico-critique, Joseph Ratzinger en laisse de côté tous les résultats gênants pour la dogmatique catholique, en la contournant habilement avec des citations des Pères de l'Église et de la liturgie. Sa vision de Jésus “ d'en haut » est inspirée de manière décisive par le dogme des conciles hellénistiques des $\mathrm{IV}^{\mathrm{e}}$ et

33 Ibid, p. 267.

34 H. Küng s'explique sur ces divergences et controverses dans ses deux volumes de Mémoires, Mon combat pour la liberté et Une vérité contestée, respectivement publiés à Paris, au Cerf, en 2006 et 2010. 
$\mathrm{v}^{\mathrm{e}}$ siècles, et par la théologie d'Augustin et de Bonaventure. Non sans entrer dans des cercles vicieux, il interprète les Évangiles synoptiques à partir de l'Évangile de Jean, et celui-ci à partir du concile de Nicée (325), que pour ma part je mesure à l'aune du Nouveau Testament. Ainsi présente-t-il constamment une conception de Jésus très divinisée, tandis que je fais ressortir le Jésus historique et ses conflits de fond dramatiques avec la hiérarchie religieuse et la piété pharisienne, avec toutes leurs conséquences. (...) Conclusion: celui qui cherche dans le Nouveau Testament le Christ dogmatique, qu'il lise Ratzinger ; celui qui cherche le Jésus historique et la proclamation des premiers chrétiens, qu'il lise Küng. » ${ }^{35}$

Situation inédite que l'écriture d'une œuvre en trois volumes sur Jésus de Nazareth débutée par le cardinal Joseph Ratzinger et achevée par le pape Benoît XVI. Celui-ci a sans doute été sincère en expliquant qu'il n'écrivait pas au nom du Magistère mais en tant qu'intellectuel chrétien soucieux de participer au débat autour de Jésus. Mais la position est délicate à tenir, quelque peu comme si Charles de Gaulle avait écrit un ouvrage sur les institutions de la France, entamé en 1957 mais terminé dans les années 1960 alors qu'il est devenu le premier président de la $V^{e}$ République. Même s'il avait affirmé n'écrire qu’à titre de simple citoyen soucieux d'apporter sa pierre au débat institutionnel, il aurait été lu comme le fondateur du nouveau régime. Ceci est d'autant plus vrai pour le Jésus de Nazareth de Joseph Ratzinger-Benoît XVI que ce dernier a souhaité réagir contre une tradition historico-critique jugée trop prégnante et oublieuse de l'essentiel de la personnalité et de l'action du Christ. Ce positionnement, clairement assumé, a donné lieu à de nombreuses réactions allant de l'approbation à la franche hostilité qui ont fait de cette publication un moment important dans l'historiographie autour de Jésus. Biographie impossible, certainement pas. Biographie à enjeux, certainement.

\author{
Philippe Foro \\ Université Toulouse - Jean Jaurès \\ 6, impasse Marcel Pendanx \\ 31100 Toulouse-France \\ philippe.foro@wanadoo.fr
}

